\title{
The quality of contemporary records made by handwriting. Comparative analysis over the period of nineteen years
}

\author{
IWONA ZIENIEWICZ \\ Department of Forensic Sciences \\ Faculty of Law, Administration, and Economics \\ University of Wroclaw, Poland
}

The advantage of our times is access to new electronic technologies which undoubtedly make our lives easier. One of them are the ever-improving text editors that allow us to write more efficiently, quickly, clearly, and, which is very important, to correct errors. This method for writing down texts is slowly displacing classic writing tools like a pen or ballpoint pen. This can be seen even more frequently during classes with students who, instead of taking notes manually, record them on portable computer devices. Also in professional work that requires writing things down, the basic tool is a computer equipped with a text editor or other user software. Today, ubiquitous social networking services also force their users to type on the keyboard. Computers have settled down in schools, and many, if not all pupils' and students' homework assignments are created on computer. It can be noticed that - compared to the past — even in primary education, as well as at subsequent stages of education, no great importance is currently attached to handwriting skills, to neatly kept notebooks, which in 
the initial stages of education are largely replaced by exercise books, filled in rather than written down by pupils from beginning to end.

The fact is that we write less and less, therefore the question can be asked whether, as a consequence, in the future we will not be undertaking this kind of activity at all, and hence, will there be no need to acquire handwriting skills, since even instead of a handwritten signature we will use its electronic substitute on a common basis? One should hope that this will happen in the distant future, which can be confirmed by the results of a survey conducted among students. Out of 56 people, 51 of them stated that they put both their signatures and produce continuous records of a private character, and in connection with their studies. Two people indicated that they make only private handwritten records and three said that they write by hand only for educational purposes. For 10 people, private records constitute only signatures. The records produced in connection with their university education in the form of signatures and continuous records are made by as many as 49 people. Nevertheless, all the respondents write more often on the computer than by hand.

An impulse to address the problem formulated in the topic of the publication are long-term observations of students' handwriting, which allowed for an assumption that the quality of handwriting is systematically decreasing, and this state of affairs may affect the possibility to identify people by their handwriting. It is commonly known that the basis for handwriting analyses are the assumptions regarding individuality and relative invariability of graphism based on the emergence of writing habits, which in turn is conditioned by the long-term process of learning how to write and improving this skill by writing a lot and often. Only then, can different handwriting features have a chance to consolidate and acquire the attribute of repeatability in various records made by the same author. In a situation when this activity is undertaken less frequently, the said process will not end with sufficient individualisation of graphism.

In order to verify the accuracy of the above thesis, the examination works written by third year law students from the years 1999-2001 and 2016-2017 were analysed. The groups compared include 25 women and 25 men of a similar age, between 20 and 25 years of age (50 handwriting samples from the years 2016-2017 and 50 handwriting samples 
from the years 1999-2001). It is assumed that handwriting in the specified age bracket has already stabilised and can be called mature.

The subject-matter of the research were the features of handwriting which testify to the level of mastery of handwriting technique. They include:

I. Handwriting class - handwriting development level:

1. well-developed handwriting,

2. medium-developed handwriting,

3. undeveloped or underdeveloped handwriting.

II. Handwriting legibility:

1. legible,

2. medium-legible,

3. illegible or with decreased legibility.

III. Handwriting neatness:

1. neat handwriting,

2. medium-neat handwriting,

3. messy handwriting.

The evaluation of handwriting development was possible by taking into account individual identification features of graphism belonging to their different groups listed in the Catalogue of Graphic Handwriting Features.

Taking into consideration the hints offered in The Dictionary of Handwriting Terms [PL: Słownik terminów pismoznawczych], the following types of features were incorporated:

1. Uniformity of the handwritten record taking into account its constancy or variability:

- handwriting size,

- angular sizes,

- layout on the baseline.

2. Departure from the school model taking into account:

- the presence of simplified graphic signs and the connecting strokes between them, as well as its elements,

- the presence of complex graphic forms as compared to the calligraphic model.

3. The presence of repetitive forms (varieties of homophonous signs and repetitive features independent of the sound of the graphic signs).

4. Steadiness of pen pressure. 
5. Handwriting smoothness level.

6. Handwriting impulse (its connectivity level).

Results of the conducted analyses

Table 1. Total handwriting development

\begin{tabular}{|c|c|c|c|}
\hline & $\begin{array}{c}\text { Well-developed } \\
\text { handwriting }\end{array}$ & $\begin{array}{c}\text { Medium- } \\
\text { developed } \\
\text { handwriting }\end{array}$ & $\begin{array}{c}\text { Undeveloped or } \\
\text { underdeveloped } \\
\text { handwriting }\end{array}$ \\
\hline Years 2016-2017 & 4 individuals & 22 individuals & 24 individuals \\
\hline Years 1999-2001 & 11 individuals & 16 individuals & 23 individuals \\
\hline
\end{tabular}

Analysing the above data, it can be concluded that the level of handwriting development in the records from the years 2016-2017 is lower than those produced in the years 1999-2001. This is most evident with regard to well-developed handwriting, of which only 4 were noted in the records from the years 2016-2017, and as many as 11 in the years 19992001. As for undeveloped handwriting, this amount is at a similar level.

Table 2. Handwriting development — women

\begin{tabular}{|c|c|c|c|}
\hline & $\begin{array}{c}\text { Well-developed } \\
\text { handwriting }\end{array}$ & $\begin{array}{c}\text { Medium- } \\
\text { developed } \\
\text { handwriting }\end{array}$ & $\begin{array}{c}\text { Undeveloped or } \\
\text { underdeveloped } \\
\text { handwriting }\end{array}$ \\
\hline Years 2016-2017 & 4 individuals & 15 individuals & 6 individuals \\
\hline Years 1999-2001 & 6 individuals & 7 individuals & 12 individuals \\
\hline
\end{tabular}

The results of observations of the women's handwriting are not that unambiguous, and they demonstrate a tendency different from the one in the men's handwriting. The amount of well-developed handwriting in women remains at a similar level in the compared periods, whereas interestingly, more cases of undeveloped handwriting were noted in the records produced in the years 1999-2001. 
Table 3. Handwriting development — men

\begin{tabular}{|c|c|c|c|}
\hline & $\begin{array}{c}\text { Well-developed } \\
\text { handwriting }\end{array}$ & $\begin{array}{c}\text { Medium- } \\
\text { developed } \\
\text { handwriting }\end{array}$ & $\begin{array}{c}\text { Undeveloped or } \\
\text { underdeveloped } \\
\text { handwriting }\end{array}$ \\
\hline Years 2016-2017 & - & 7 individuals & 18 individuals \\
\hline Years 1999-2001 & 5 individuals & 9 individuals & 11 individuals \\
\hline
\end{tabular}

The biggest changes regarding a decrease in the handwriting development level are visible in the men's records. None of the graphisms produced in the years 2016-2017 can be evaluated as well-developed, while the amount of undeveloped handwritings is by far higher than in the graphisms from the years 1999-2001.

Table 4. Total handwriting legibility

\begin{tabular}{|c|c|c|c|}
\hline & $\begin{array}{c}\text { Legible } \\
\text { handwriting }\end{array}$ & $\begin{array}{c}\text { Medium-legible } \\
\text { handwriting }\end{array}$ & $\begin{array}{c}\text { Handwriting } \\
\text { with decreased } \\
\text { legibility } \\
\text { (illegible) }\end{array}$ \\
\hline Years 2016-2017 & 22 individuals & 19 individuals & 9 individuals \\
\hline Years 1999-2001 & 30 individuals & 17 individuals & 3 individuals \\
\hline
\end{tabular}

As the conducted analysis shows, a serious problem in contemporary records is producing legible records. There are a lot more illegible records in the graphisms from the years 2016-2017 than in those produced in the years 1999-2001.

Table 5. Handwriting legibility — women

\begin{tabular}{|c|c|c|c|}
\hline & $\begin{array}{c}\text { Legible } \\
\text { handwriting }\end{array}$ & $\begin{array}{c}\text { Medium-legible } \\
\text { handwriting }\end{array}$ & $\begin{array}{c}\text { Handwriting } \\
\text { with decreased } \\
\text { legibility } \\
\text { (illegible) }\end{array}$ \\
\hline Years 2016-2017 & 17 individuals & 7 individuals & 1 individual \\
\hline Years 1999-2001 & 17 individuals & 6 individuals & 2 individuals \\
\hline
\end{tabular}


The above regularity has not been noted in the women's handwriting since the amount of legible, medium-legible and illegible records is at a similar level.

Table 6. Handwriting legibility — men

\begin{tabular}{|c|c|c|c|}
\hline & $\begin{array}{c}\text { Legible } \\
\text { handwriting }\end{array}$ & $\begin{array}{c}\text { Medium-legible } \\
\text { handwriting }\end{array}$ & $\begin{array}{c}\text { Handwriting } \\
\text { with decreased } \\
\text { legibility } \\
\text { (illegible) }\end{array}$ \\
\hline Years 2016-2017 & 5 individuals & 12 individuals & 8 individuals \\
\hline Years 1999-2001 & 13 individuals & 11 individuals & 1 individual \\
\hline
\end{tabular}

Similarly to the handwriting development aspect, there is a decreasing trend in the men's handwriting legibility, as shown in the table above, the records produced in the years 1999-2001 are far more legible.

Table 7. Total handwriting neatness

\begin{tabular}{|c|c|c|c|}
\hline & Neat handwriting & $\begin{array}{c}\text { Medium-neat } \\
\text { handwriting }\end{array}$ & $\begin{array}{c}\text { Messy } \\
\text { handwriting }\end{array}$ \\
\hline Years 2016-2017 & 5 individuals & 23 individuals & 22 individuals \\
\hline Years 1999-2001 & 8 individuals & 27 individuals & 15 individuals \\
\hline
\end{tabular}

Table 8. Handwriting neatness - women

\begin{tabular}{|c|c|c|c|}
\hline & Neat handwriting & $\begin{array}{c}\text { Medium-neat } \\
\text { handwriting }\end{array}$ & $\begin{array}{c}\text { Messy } \\
\text { handwriting }\end{array}$ \\
\hline Years 2016-2017 & 5 individuals & 14 individuals & 6 individuals \\
\hline Years 1999-2001 & 6 individuals & 11 individuals & 8 individuals \\
\hline
\end{tabular}

Table 9. Handwriting neatness - men

\begin{tabular}{|c|c|c|c|}
\hline & Neat handwriting & $\begin{array}{c}\text { Medium-neat } \\
\text { handwriting }\end{array}$ & $\begin{array}{c}\text { Messy } \\
\text { handwriting }\end{array}$ \\
\hline Years 2016-2017 & - & 9 individuals & 16 individuals \\
\hline Years 1999-2001 & 2 individuals & 16 individuals & 7 individuals \\
\hline
\end{tabular}


As the above data show that handwriting neatness has also deteriorated. This tendency is more evident in the men's handwriting, as compared to the women's graphism, in whom this feature is more stabilised.

\section{Final conclusions}

1. The conducted analysis of the records shows a deteriorating trend in the handwriting quality of contemporary records. The decreasing trend in this scope is clearly visible in men's handwriting. It is not that obvious in women's handwriting.

2. This status quo can cause difficulties in identification of the record's author, since undeveloped or underdeveloped handwriting - due to the absence of individualised features - is a more difficult examination material which has not been sufficiently developed and consolidated the writing habits, illustrated by several examples of the examined records which - frankly speaking - resemble school handwriting.

3. Decreased handwriting legibility and neatness found in a number of examined graphisms can make it impossible to analyse the features which cannot be distinguished in such cases.

4. In numerous handwriting samples, the inability to produce a uniform, legible and neat record can be a result of less and less frequent writing practice. These records do not look like typical school handwriting, as they have had enough time to modify. A departure from the calligraphic model frequently has a form of far-reaching simplifications which make it impossible to read the text, at least partially. Such examples of graphism also show handwriting disturbances including: irregular size, inclination, or lack of smoothness in the graphic lines which can be caused by the aforementioned absence of writing activity. This phenomenon can testify to the regression in handwriting skills.

\section{References}

Koziczak A., Metody pomiarowe w badaniach pismoznawczych, Kraków 1997, pp. 35-37. Stownik terminów pismoznawczych [The Dictionary of Handwriting Terms], http://prawouam-stp.home.amu.edu.pl/ (access: 21.10.2017). 


\section{Summary}

Handwriting is a method to express and preserve thoughts by means of graphic signs. In the investigative and judicial practice, it is treated as an individual creation used for identification purposes in case of doubts over the authenticity of a written record. Nowadays, however, writing by hand is not the only way of conveying information, which results in moving away from this traditional method in favour of modern solutions. In the future, this tendency may limit the possibility to identify the author of a record on the basis of a handwritten record. Therefore, the purpose of this publication is to check whether contemporary handwritings show any signs of deterioration in the quality of handwritten records.

Keywords: handwriting, features of handwriting, handwriting analyses. 


\section{Example of contemporary undeveloped handwriting I Unformed handwriting}

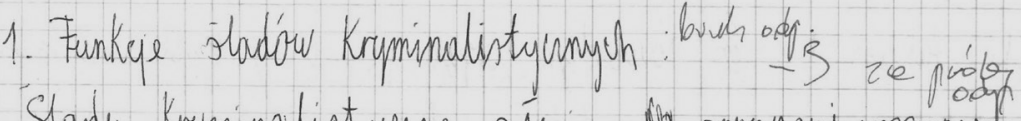

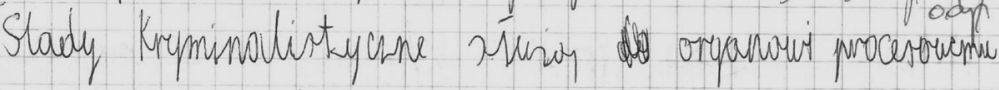

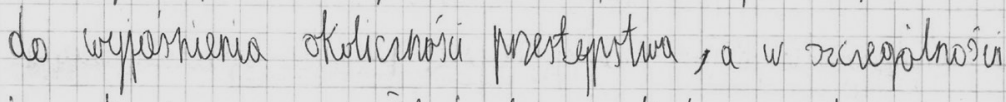

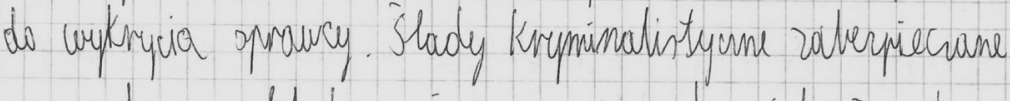

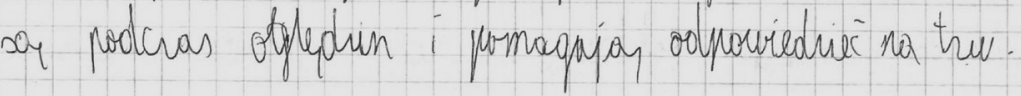

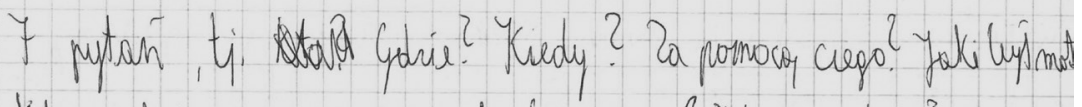

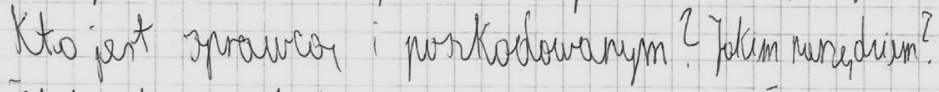

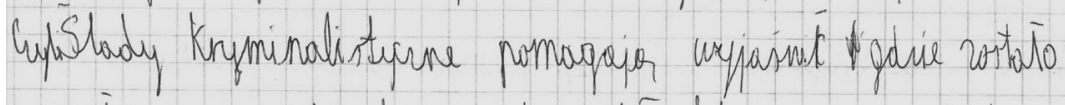

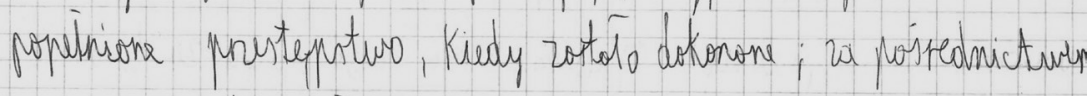

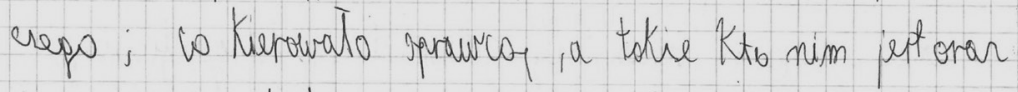
Kim pat porkodowitany.

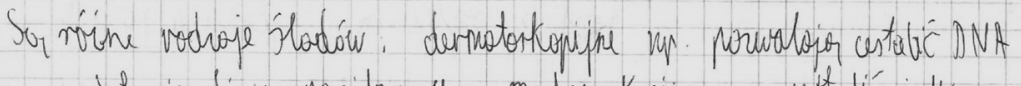

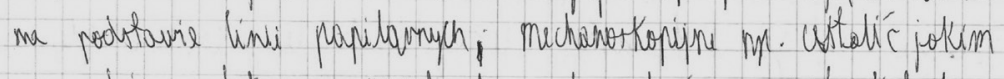

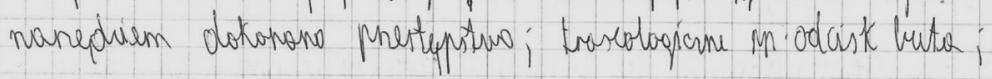

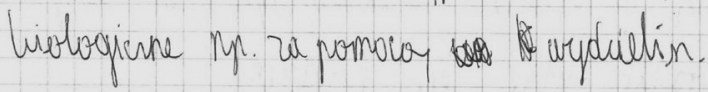

Undeveloped handwriting, school handwriting 


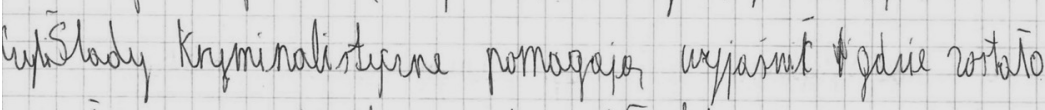

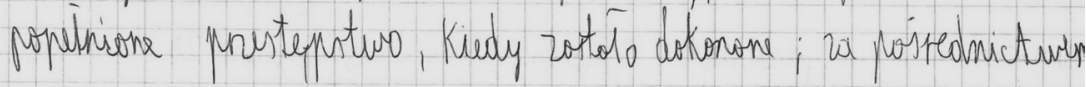

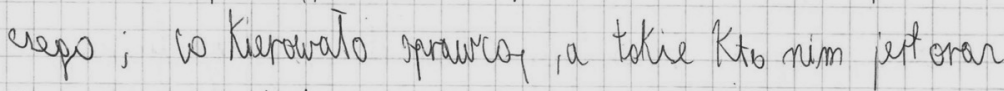
kim jut porktodoranes.

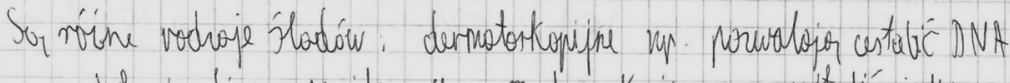

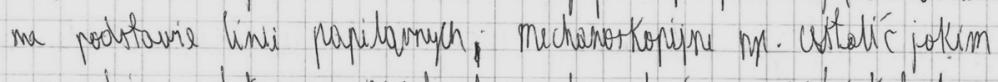

Irregular handwriting size and its inclination

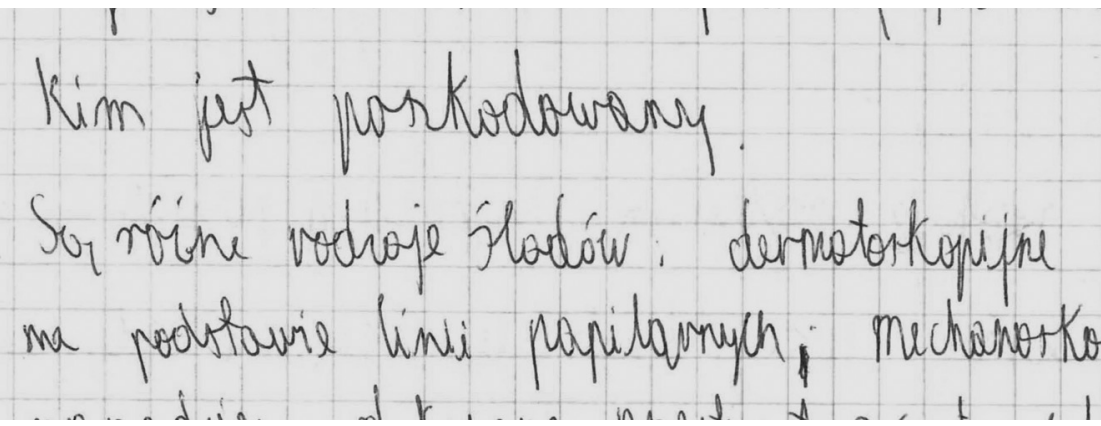

Decreased handwriting smoothness

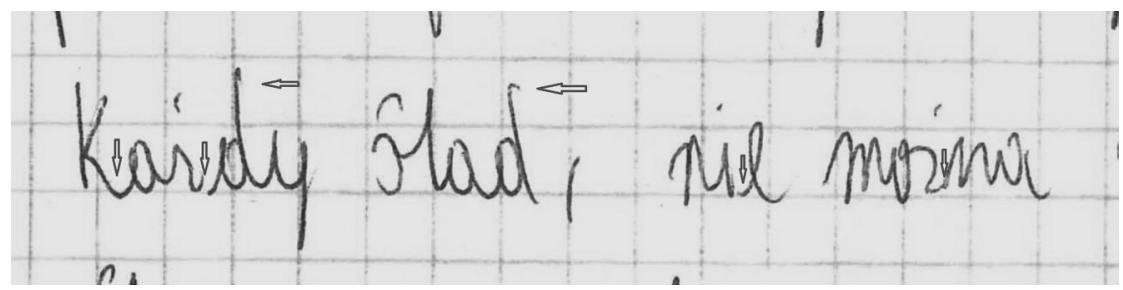

Irregular handwriting shading 


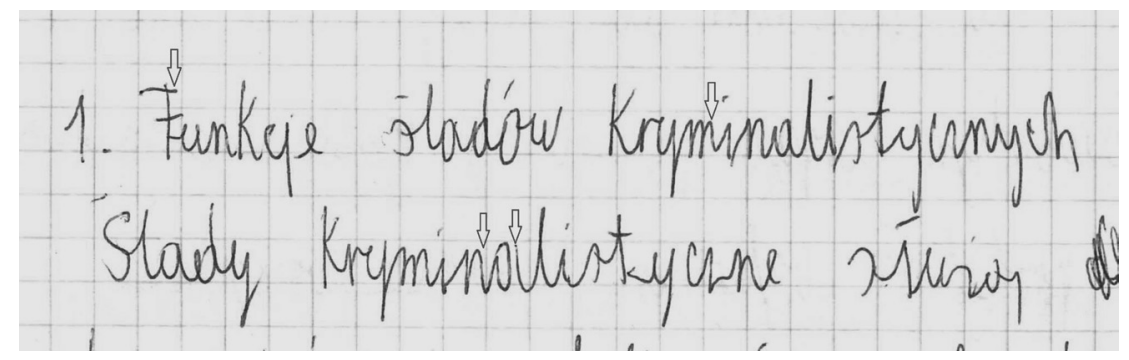

Prevailing letter impulse (gramm impulse), sometimes in two and three letters

\section{Example of contemporary undeveloped handwriting II Example of regressed handwriting skills}

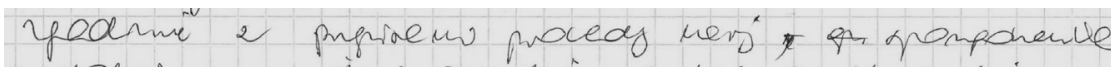

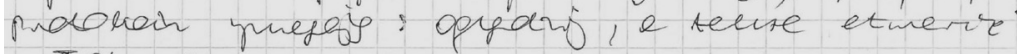

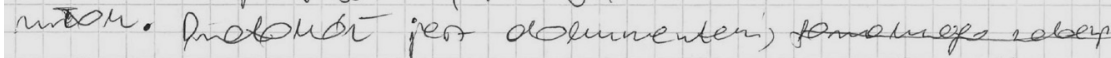

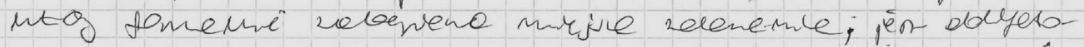

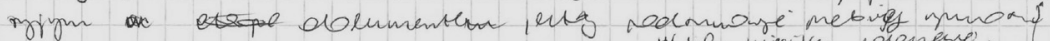

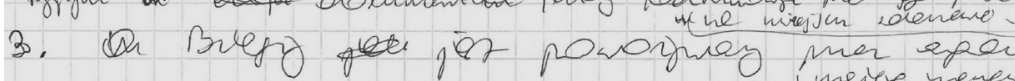

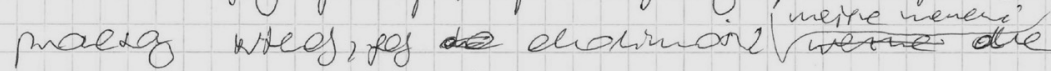

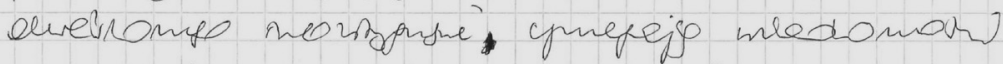

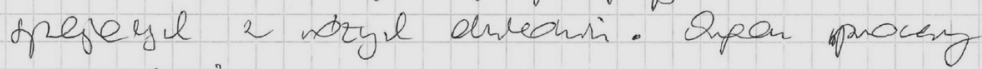
- poolve" a pupromi procedy nery-paraze. lorite wisolgy ponenameure?

Brogy a po pupromedreun reederi prou pole

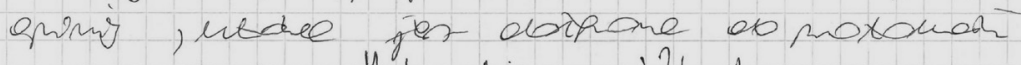

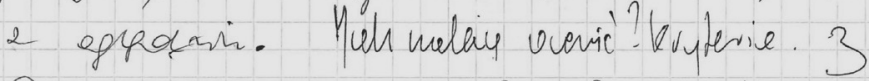
orper procenag uraghfé th olocké

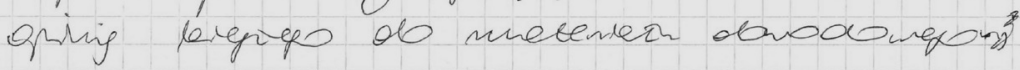

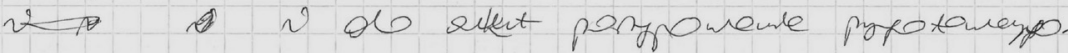
Opinite lexpiefo pert dowodeur oxdogmy Underdeveloped, modified handwriting 


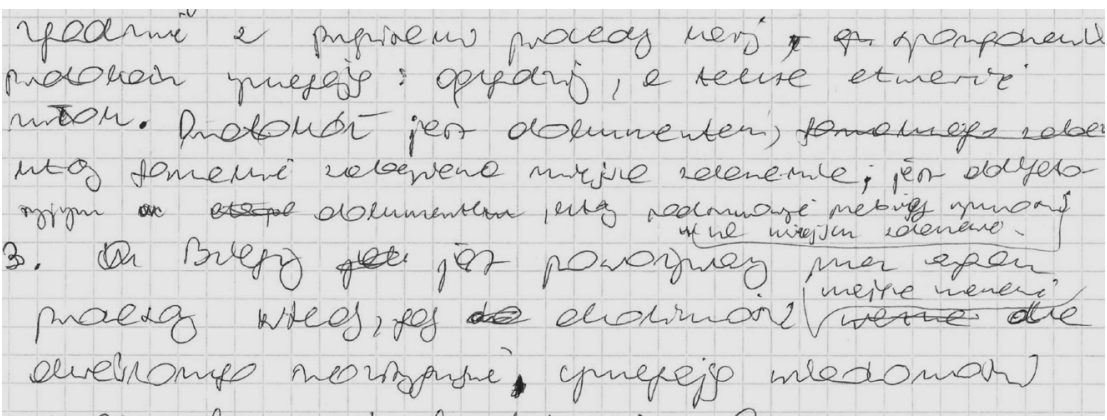

Irregular handwriting size and inclination. Considerably decreased handwriting legibility

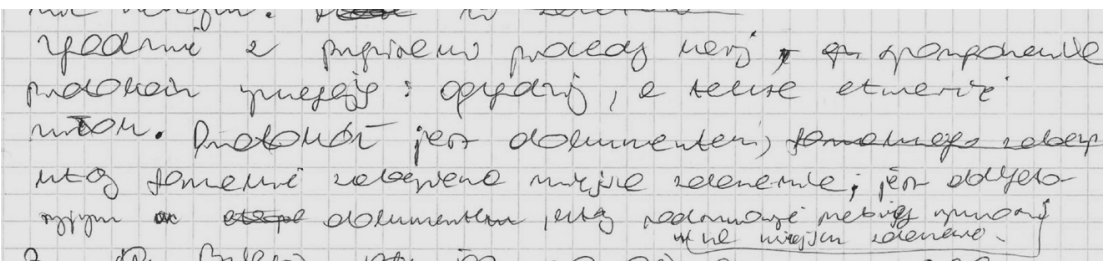

Irregular arrangement of the record on the baseline 